\title{
Development of a novel model
} of cholecystectomy in subsequently ovariectomized mice and characterization of metabolic and gastrointestinal phenotypes: a pilot study

Celeste Alexander ${ }^{1 *}$, Tzu-Wen L. Cross' ${ }^{1}$, Anne H. Lee ${ }^{2}$, Lindsey K. Ly ${ }^{1}$, Miranda D. Vieson ${ }^{3}$, Jason M. Ridlon 1,2,4,5, Erik R. Nelson ${ }^{1,4,5,6,7}$ and Kelly S. Swanson ${ }^{1,2^{*}}$

\begin{abstract}
Background: Cholecystectomy (XGB) is the most common abdominal surgery performed in the United States and is associated with an increased post-surgery incidence of metabolic and gastrointestinal (GI) diseases. Two main risk factors for XGB are sex (female) and age (40-50 yr), corresponding with onset of menopause. Post-menopausal estrogen loss alone facilitates metabolic dysfunction, but the effects of XGB on metabolic and Gl health have yet to be investigated in this population. Study objectives were to (1) identify possible short-term effects of XGB and (2) develop a novel murine model of $X G B$ in human menopause via subsequent ovariectomy $(O V X)$ and assess longitudinal effects of OVX on metabolism, GI physiology, and GI microbiota in XGB mice.

Methods: Female C57BL/6 mice were utilized in two parallel studies (S1\&S2). In S1, XGB mice were compared to a non-XGB baseline group after six wk. In S2, mice were XGB at wk0, either sham (SHM) or OVX at wk6, and sacrificed at wk12, wk18, and wk24. Body composition assessment and fresh fecal collections were conducted periodically. Serum and tissues were collected at sacrifice for metabolic and GI health endpoints.

Results: Compared to baseline, XGB increased hepatic CYP7A1 and decreased HMGCR relative expression, but did not influence BW, fat mass, or hepatic triglycerides after six wk. In S2, XGB/OVX mice had greater BW and fat mass than XGB/SHM. Cecal microbiota alpha diversity metrics were lower in XGB/OVX mice at wk24 compared the XGB/ SHM. No consistent longitudinal patterns in fasting serum lipids, fecal microbial diversity, and Gl gene expression were observed between $\mathrm{S} 2$ groups.
\end{abstract}

Conclusions: In addition to developing a novel, clinically-representative model of XGB and subsequent OVX, our results suggest that OVX resulted in the expected phenotype to some extent, but that XGB may modify or mask some responses and requires further investigation.

Keywords: Gallbladder removal, Menopause, Microbiome, Metabolism

*Correspondence: calxndr3@illinois.edu; ksswanso@illinois.edu ${ }^{1}$ Division of Nutritional Sciences, University of Illinois at UrbanaChampaign, 1207 W Gregory Dr, Urbana, IL 61801, USA

Full list of author information is available at the end of the article

\section{Introduction}

Cholecystectomy (XGB) is the surgical removal of the gallbladder due to disease-most commonly symptomatic cholelithiasis (gallstone formation) and 
gallbladder cancer-and is the most common abdominal surgery performed in the United States, with over 800,000 procedures performed each year and estimated costs exceeding $\$ 6$ billion annually $[1,2]$. Individuals who undergo XGB are typically around 49 years of age (median), female (70.7\%), Caucasian (65.5\%), and overweight or obese (74.2\%) [3]. Somewhat paradoxically, given that the median age corresponds to perimenopausal or post-menopausal women, estrogen increases the likelihood of gallstone formation in preclinical models [4]. However, estrogen replacement therapy following menopause has been associated with an increased risk of XGB, possibly explaining, at least in part, the greater rate of XGB in females [5-7].

Following XGB, bile is continuously released into the duodenum as it is made by the liver, regardless of fed or fasted state, chronically exposing the small intestine to small volumes and concentrations of bile acids (BA). Although some post-XGB individuals report symptoms of impaired dietary fat digestion following surgery, studies have not shown significant impairments in fat absorption [8,9]. Although some follow-up studies have led to the belief that XGB does not result in any long-term medical consequences $[10,11]$, more recent epidemiological and clinical studies suggest that XGB may increase the risk of developing diseases including metabolic syndrome [12], non-alcoholic fatty liver disease [13-17], cardiovascular disease [18], GI cancers [19-22], small intestinal bacterial overgrowth [23, 24], and bile reflux [25]. XGB may also alter the composition of the fecal microbiota in human subjects [26-29]. Similar to findings in humans, XGB mouse studies have reported an accumulation of hepatic triglycerides (TG), increased fasting serum TG, and increased very low-density lipoprotein production compared to nonXGB controls [30, 31]. Collectively, these studies suggest perturbations in metabolic and gastrointestinal (GI) health following XGB, though there is still a need for additional pre-clinical studies in appropriate animal models and prospective clinical trials to confirm these findings.

It is hypothesized that the continuous flow of bile into the GI tract after XGB not only has impacts locally, but also leads to systemic health perturbations. This is because BA act as potent signaling molecules that regulate their own production, inflammatory responses, and energy metabolism [32]. Furthermore, XGB could potentially allow either blooms of bile-intolerant bacteria in the absence of larger volumes of concentrated bile that normally curbs growth or blooms of bile-tolerant bacteria. Some colonic bacteria are also capable of altering the BA pool by metabolizing BA [33]. Theoretically, XGB may result in compositional or functional changes to the GI microbiome, which in turn alters BA pool composition and subsequent metabolic signaling.

Few studies investigating metabolic changes post-XGB have been performed in animal models to date. Despite the fact that over $70 \%$ of XGB patients are female, recent research has only utilized male animal models [2, 30, 31]. While male XGB mice may provide some insight not possible in human studies, differences in sex hormones are known to greatly influence both host metabolism and the GI microbiome [34]. The median age of individuals undergoing XGB corresponds to the onset of menopause in women $[3,35]$. Because menopause is known to exacerbate metabolic dysfunction, it can be argued that studying XGB in an intact, pre-menopausal animal model would not be sufficiently representative of the main clinical population [35]. One of the most commonly used and validated models of menopause in rodents is ovariectomy (OVX), the surgical removal of the ovaries. The current lack of understanding about the impact of XGB on metabolism and GI health and microbiota in middleaged women is of clinical concern, given the number of procedures performed each year and evidence suggesting consequent negative effects. Thus, the purpose of this study was to develop a novel model of XGB in subsequently OVX mice to more closely represent the main XGB patient population, and characterize the resulting metabolic and GI phenotypes.

\section{Methods}

\section{Animals, diet, and study design}

All procedures were approved by the University of Illinois Institutional Animal Care and Use Committee before the study (protocol \#17040) and were performed in accordance with the United States Public Health Service Policy on Humane Care and Use of Laboratory Animals. Fortyeight eight-wk-old female C57BL/6 mice (Jackson Laboratory, Bar Harbor, ME) were used in two studies run in parallel. All mice were fed a high-fat $(45 \% \mathrm{kcal}$ from fat, HFD), low-sucrose (7\% kcal) diet (Table 1) for the duration of the studies (6 or $24 \mathrm{wk}$ ) and housed in an environmentally-controlled facility with a $12 \mathrm{~h} \mathrm{light} / 12 \mathrm{~h}$ dark cycle. Mice were given ad libitum access to food and intake was measured twice weekly. Mice were given access to fresh water at all times. Body weight (BW) was measured weekly throughout the study.

The study timelines are presented in Fig. 1. The aim of Study 1 (S1) was to identify relatively short-term (six wk) effects of XGB on study outcomes by comparing mice to a baseline group without surgical alterations. The aim of Study 2 (S2) was to develop a novel model of XGB in OVX mice and to characterize the resulting metabolic and GI phenotypes compared to XGB mice with intact ovaries (sham OVX operation, SHM). Mice were 
Table 1 Ingredient and macronutrient composition of the experimental diet

\begin{tabular}{lc}
\hline Ingredients & \% as-fed (g/100 g) \\
\hline Casein, 80 Mesh & 23.307 \\
Lard & 20.685 \\
Corn starch & 20.347 \\
Maltodextrin 10 & 11.654 \\
Sucrose & 8.274 \\
Cellulose, BW200 & 5.827 \\
Corn Oil & 2.913 \\
Potassium citrate, $1 \mathrm{H}_{2} \mathrm{O}$ & 1.923 \\
Dicalcium phosphate & 1.515 \\
Mineral mix S10026 & 1.165 \\
Vitamin mix V10001 & 1.165 \\
Calcium carbonate & 0.006 \\
L-cystine & 0.003 \\
Choline bitartrate & 0.002 \\
\hline Macronutrient composition & $\mathbf{k c a l}$ \\
\hline Fat & 45 \\
Carbohydrate & 35 \\
Protein & 20 \\
\hline Gross energy (kcal/g) & 4.73 \\
\hline
\end{tabular}

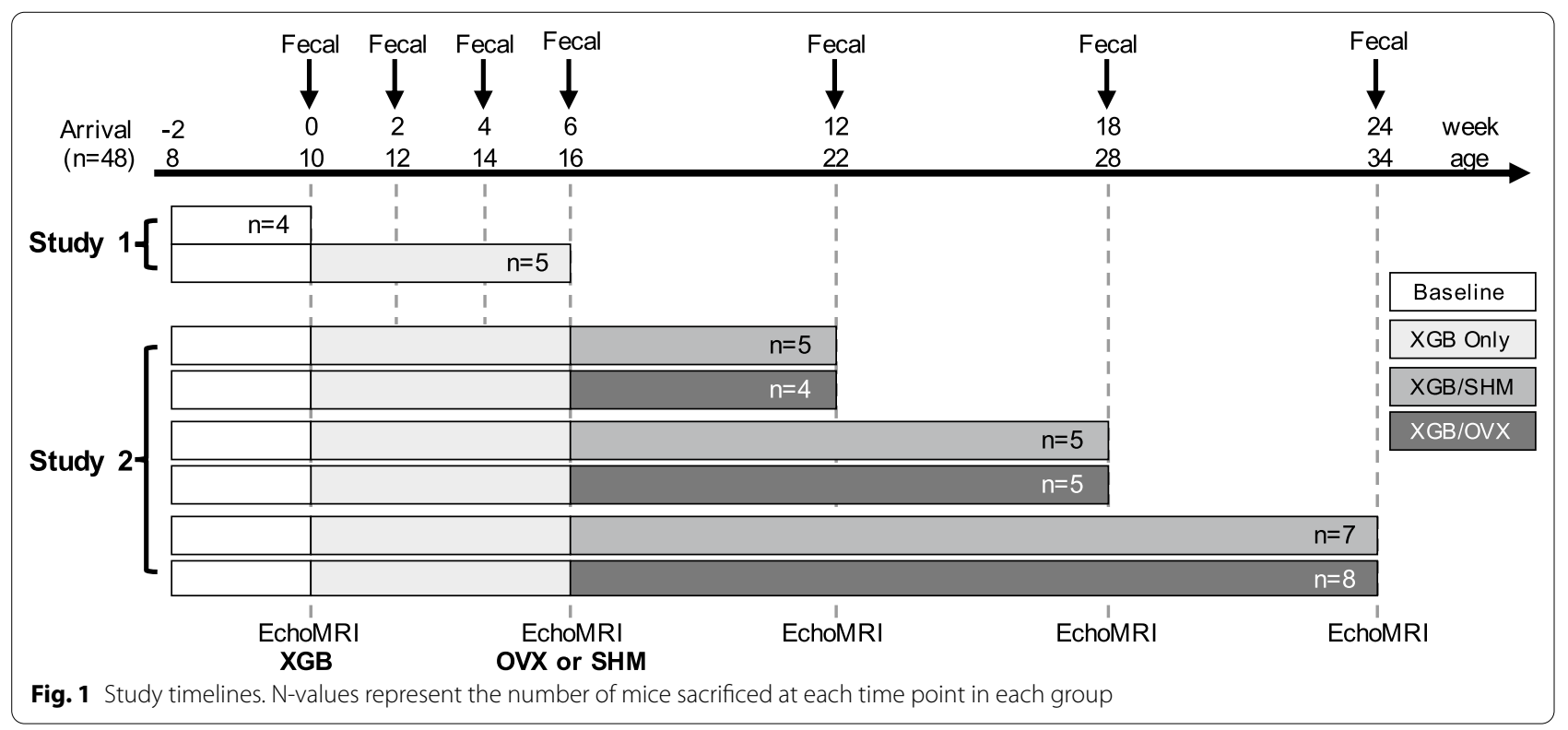

group-housed ( $n=4 /$ cage) and adapted to the experimental diet and facilities for two wk upon arrival. To reduce variability in baseline microbiota, bedding from all cages was mixed and redistributed every $4-5 \mathrm{~d}$ during the adaptation period. After adaptation, mice $(\mathrm{n}=4$, ten-wk-old) were euthanized by $\mathrm{CO}_{2}$ inhalation for $\mathrm{S} 1$ baseline (wk 0 ) measurements and all remaining 44 mice (ten-wk-old) underwent XGB at wk 0 and were housed individually in standard shoebox cages for the remainder of the studies. In S1, mice were euthanized six wk post-XGB $(n=5)$ for tissue collection. In S2, mice were randomly assigned to undergo either SHM or OVX procedures on wk 6 (16-wk-old), resulting in two experimental groups: XGB/SHM and XGB/OVX. OVX was 
performed following XGB in order to mimic progression into menopause. Mice from each group in S2 were euthanized at wk 12, 18, and 24 for tissue collection and longitudinal analyses. In both studies, all existing mice were scanned using an EchoMRI-700 (Echo Medical Systems, Houston, TX) for body composition analysis on wk 0, 6, 12 , 18, and 24. Fresh fecal samples were collected for S2 at wk $6,12,18$, and 24 and flash frozen for microbiota analysis.

\section{Surgical procedures}

Hair was removed at the site of operation. Mice were fasted overnight prior to surgeries. Mice were anesthetized with isoflurane $(1 \mathrm{~L} / \mathrm{min} \mathrm{O} 2,4 \%$ isoflurane reduced to $2 \%$ after anesthetized, Phoenix Pharmaceuticals, Burlingame, CA) throughout the procedures. Preparatory and surgical tasks were split between two people to maintain a sterile environment. Once under anesthesia, Puralube ${ }^{\circledR}$ (Dechra, Northwich, United Kingdom) was applied to eyes to counter-act the reduction in tearing. Mice were then injected (SQ) with $5 \mathrm{mg} / \mathrm{kg}$ carprofen (nonsteroidal anti-inflammatory, Zoetis, Parsippany-Troy Hills, NJ). The site of operation was sterilized with Povidone/Iodine swabs (Med Vet International, Mettawa, IL) and ethanol swabs three times.

\section{$X G B$ procedure}

A vertical incision was made through the skin above the liver (on abdomen), followed by a vertical incision through the abdominal muscle wall. The gallbladder was externalized, emptied, and removed using cauterizing scissors. Muscle walls and skin were sutured with absorbable sutures (VCP822G, Ethicon, Somerville, NJ). Two drops of bupivacaine $(0.25 \%$, SKM Pharma Pvt Ltd., Bengaluru, India) was applied on top of the muscle wall. Medbond tissue adhesive (CP Medical, Norcross, GA) was applied to the closed incision.

\section{OVX procedure}

A vertical incision was made through the skin above the ovary fat pad (flank), followed by a horizontal incision through the abdominal muscle wall. The ovary was externalized and removed using cauterizing scissors. The fat pad was relocated back in the peritoneal cavity, and muscle walls were closed using absorbable sutures if needed. A drop of bupivacaine $(0.25 \%)$ was applied on top of the muscle wall. Wound clips (BD Autoclip ${ }^{\mathrm{TM}}$ Wound Closing System, $9 \mathrm{~mm}$, BD, Franklin Lakes, NJ) were placed on the incision. This was repeated for the other ovary. The SHM procedure was identical, except the ovaries were not removed.

After both procedures, carprofen was administered $(5 \mathrm{mg} / \mathrm{kg})$ in all mice at $24 \mathrm{~h}$ and $48 \mathrm{~h}$ post-surgery.
Carprofen was administered again at $72 \mathrm{~h}$ only if pain or discomfort was observed. Wound clips were removed at 10-14 d post-surgery. Mice typically did not exhibit visible signs of pain or distress after $24-48 \mathrm{~h}$ post-surgery.

\section{Serum collection and analysis}

Blood samples were collected immediately after euthanasia before dissection via cardiac puncture, placed in sterile microcentrifuge tubes, and allowed to clot at room temperature for $30 \mathrm{~min}$ prior to centrifugation for $10 \mathrm{~min}$ at $2000 \mathrm{~g}$ and $4{ }^{\circ} \mathrm{C}$ and storage at $-80^{\circ} \mathrm{C}$. Serum samples were sent to a commercial laboratory to determine TG and total cholesterol concentrations (Comparative Clinical Pathology Services, Columbia, MO) using an Olympus AU680 automated chemistry analyzer (Beckman-Coulter, Brea, CA).

\section{Adipose depots, hepatic lipidosis, and hepatic TG quantification}

Four major adipose depots-subcutaneous, gonadal, mesenteric, perirenal-were removed at sacrifice and weighed. For pathology scoring, liver samples were fixed in 10\% neutral-buffered formalin for at least $24 \mathrm{~h}$ then stored in $80 \%$ ethanol indefinitely. Samples were trimmed and paraffin-embedded prior to being sliced and stained with H\&E at the Veterinary Diagnostic Lab at the University of Illinois. Hepatic lipidosis was scored by a blinded pathologist (MDV) at the University of Illinois. Detailed criteria for lipidosis scoring are presented in Table 2. For quantification of TG, liver samples were flash frozen upon collection and stored at $-80{ }^{\circ} \mathrm{C}$ prior to extraction. Sample extraction was adapted from previously described methods [36]. Briefly, $30 \mathrm{mg}$ of whole liver was added to $1 \mathrm{ml}$ of chloroform-methanol (2:1 vol/ vol), homogenized for 2 min using a TissueLyser LT (Qiagen, Hilden, Germany), and gently agitated overnight at $4{ }^{\circ} \mathrm{C}$. One $\mathrm{mL}$ of $4 \mathrm{mM} \mathrm{MgCl}$ was added, vortexed, and centrifuged for $1 \mathrm{~h}$ at $1000 \mathrm{~g}$ at $4{ }^{\circ} \mathrm{C}$. The organic phase was removed, evaporated overnight, and reconstituted in $500 \mathrm{uL}$ tert-butanol-Triton X-114 mix $(3: 2 \mathrm{vol} / \mathrm{vol})$ and vortexed. TG concentrations were then determined using a commercially available kit (\#T2449 \& \#F6428, Sigma, St. Louis, MO) with a spectrophotometer (Synergy HT, BioTek, Winooski, VT).

\section{RNA extraction, RT-PCR, and quantitative PCR}

Total RNA was extracted from liver, distal ileum, and distal colon samples using the RNeasy Mini Kit (Qiagen, Hilden, Germany). Conversion to cDNA was performed using SuperScript III Reverse Transcriptase (Invitrogen, Carlsbad, CA). Genes related to energy and cholesterol metabolism, BA synthesis, signaling, and transport, and barrier function were measured via real-time qPCR using 
Table 2 Hepatic lipidosis scoring criteria

\begin{tabular}{|c|c|}
\hline Score & Criteria \\
\hline 0 (Normal) & $<3$ macrovesicular lipid vacuoles per lobule \\
\hline 1 (Minimal) & 5-10\% hepatocytes affected, midzonal location, no hepatocyte swelling, macrovesicular lipid vacuoles \\
\hline 2 (Mild) & $\begin{array}{l}\text { 10-25\% hepatocytes affected, midzonal location with some centrilobular involvement, no or mild hepatocyte swelling, mainly } \\
\text { macrovesicular lipid vacuolation with occasional microvesicular lipid vacuolation }\end{array}$ \\
\hline 3 (Moderate) & $\begin{array}{l}\text { 25-50\% hepatocytes affected, midzonal and centrilobular distribution, moderate to marked hepatocyte swelling centrilobular and } \\
\text { midzonal; minimal to mild swelling periportal, macro- and microvesicular lipid vacuoles }\end{array}$ \\
\hline 4 (Marked) & $\begin{array}{l}\text { 50-75\% hepatocytes affected, midzonal, centrilobular and some periportal distribution, moderate to severe hepatocyte swelling, } \\
\text { macro- and microvesicular lipid vacuolation }\end{array}$ \\
\hline 5 (Severe) & $\begin{array}{l}\text { 75-95\% hepatocytes affected, midzonal, centrilobular and periportal distribution, severe hepatocyte swelling centrilobular and } \\
\text { midzonal, moderate to marked swelling periportal, macro- and microvesicular vacuolation }\end{array}$ \\
\hline 6 (Very Severe) & $\begin{array}{l}95-100 \% \text { hepatocytes affected, midzonal, centrilobular and periportal distribution, severe hepatocyte swelling all zones, macro- and } \\
\text { microvesicular vacuolation }\end{array}$ \\
\hline
\end{tabular}

a QuantStudio 7 Real-Time PCR System (Applied Biosystems, Foster City, CA). Genes of interest and associated primers sequences are listed in Table 3. Briefly, primers were designed and purchased from Fluidigm Deltagene Assays (Fluidigm Corporation, South San Francisco, CA) and IDT Inc. (Coralville, IA). SYBR Green PCR Master Mix (Invitrogen, Carlsbad, CA) was used for real-time amplification and detection with a reaction volume of 10 $\mathrm{uL}$. The thermal protocol was $2 \mathrm{~min}$ at $50{ }^{\circ} \mathrm{C}$, and $10 \mathrm{~min}$ at $95{ }^{\circ} \mathrm{C}$, followed by 40 amplification cycles of $15 \mathrm{~s}$ at $95{ }^{\circ} \mathrm{C}$ and $1 \mathrm{~min}$ at $60{ }^{\circ} \mathrm{C}$. Data analysis was performed using the QuantStudio 6 and 7 Flex Real-Time PCR System Software (Applied Biosystems, Foster City, CA). All RNA data presented were derived using the $\Delta \Delta \Delta \Delta C T$ method, assessing gene expression relative to the housekeeping gene (PPIA), and expressing data as fold change relative to baseline ( $\mathrm{wk} 0$ ).

\section{Microbiota analysis}

Cecal and fecal bacterial DNA from S2 was extracted using the PowerLyzer PowerSoil Kit (Qiagen, Hilden, Germany) with bead beating using a vortex adaptor (cat. no. 13000-V1-24, Qiagen, Hilden Germany). The concentration of extracted DNA was quantified using a Qubit ${ }^{\circledR}$
3.0 Fluorometer (Life Technologies, Carlsbad, CA). 16S rRNA gene amplicons of the V4 region were generated, pooled, and sequenced for each sample as previously described by our lab [37]. $16 \mathrm{~S}$ rRNA gene amplicons were generated using a Fluidigm Access Array (Fluidigm Corporation, South San Francisco, CA) in combination with Roche High Fidelity Fast Start Kit (Roche, Basel, Switzerland). The primers 515F (5'-GTGYCAGCMGCC GCGGTAA-3') and 806R (5'-GGACTACNVGGG TWTCTAAT-3') that target a 252-bp fragment of the $\mathrm{V} 4$ region were used for amplification (primers synthesized by IDT Inc., Coralville, IA) [38]. CS1 forward tag and CS2 reverse tag were added according to the Fluidigm protocol. Quality of the amplicons was assessed using a Fragment Analyzer (Advanced Analytics, Ames, IA) to confirm amplicon regions and sizes. A DNA pool was generated by combining equimolar amounts of the amplicons from each sample. The pooled samples were then size-selected on a $2 \%$ agarose E-gel (Life Technologies, Carlsbad, CA) and extracted using a Qiagen gel purification kit (Qiagen, Hilden, Germany). Cleaned size-selected pooled products were run on an Agilent 2100 Bioanalyzer to confirm appropriate profile and average size. Sequencing was performed at the W. M. Keck

\section{Table 3 Target genes of interest and associated primers}

\begin{tabular}{|c|c|c|}
\hline Target gene (Alias) & Forward primer $\left(5^{\prime} \rightarrow 3^{\prime}\right)$ & Reverse primer $\left(5^{\prime} \rightarrow 3^{\prime}\right)$ \\
\hline CYP7A1 & TGTGGTAGTGAGCTGTTGCATA & GGAATCAACCCGTTGTCCAA \\
\hline FGF15 & CGCGGACGGCAAGATATAC & ACAGTCCATTTCCTCCCTGAA \\
\hline$H M G C R$ & ACCAAACCCCGTAACCCAAA & AGCGACTATGAGCGTGAACA \\
\hline MGLL & GTCAATGCAGACGGACAGTAC & CATAACGGCCACAGTGTTCC \\
\hline MUC2 & CAGCACACCAACCAAAACCA & CACAGCCACCAGGTCTCATTA \\
\hline NR1H4 (FXR) & GCCTCTGGGTACCACTACAA & GTACACGGCGTTCTTGGTAA \\
\hline SLC10A1 (NTCP) & TTGCGCCATAGGGATCTTCC & TGATGACAGACAGGACTGTGAC \\
\hline SLC1OA2 (ASBT) & TGGAGGAACTGGCTCCAATA & GGAGCAAGTGGTCATGCTAA \\
\hline
\end{tabular}


Center for Biotechnology at the University of Illinois using an Illumina MiSeq using version 3 reagents (Illumina Inc., San Diego, CA).

Forward reads were trimmed using the FASTX-Toolkit (version 0.0.14) and QIIME 2.2019.4 was used to process the resulting sequence data [39]. An average of 38,896 reads per sample were obtained before processing. Briefly, high-quality (quality value $\geq 20$ ) sequence data derived from the sequencing process were demultiplexed. Data were then denoised and assembled into a feature table of amplicon sequence variants (ASV) using DADA2 [40]. Taxonomy was assigned using the SILVA 132 database [41]. An even sampling depth of 15,802 and 10,123 sequences per sample was used for assessing alpha- and beta-diversity measures for cecal and fecal samples, respectively. Alpha-diversity was estimated using observed-OTUs, Shannon's index, and Faith's PD metrics. Beta-diversity was assessed using weighted and unweighted UniFrac distance measures and presented with principal coordinates analysis (PCoA) plots [42].

\section{Statistical analysis}

Repeated measures data (S2 body weight and EchoMRI) were analyzed using the 'glimmix' procedure for repeated measures data, testing the main effects and interaction of surgery and time in SAS (version 9.4; SAS Institute). Data collected at the time of sacrifice were analyzed using either the exact Wilcoxon signed-rank test (proc npar1way) or Student's t test (proc ttest) in SAS. For microbiota sequencing data, assigned taxa that failed to reach an average relative abundance of at least $0.01 \%$ across all samples or were not detectable in at least $20 \%$ of all samples were discarded. Statistical significance was set as $p<0.05$ with $p<0.10$ considered as trends. Data are either represented as boxplots or reported as least square means with standard errors of the mean (SEM).

\section{Results}

Within six wk, XGB did not result in differences in BW (Fig. 2a) or body fat mass (Fig. 2b) compared to baseline. Over time, XGB/OVX led to greater $(p<0.001)$ weight gain compared to $\mathrm{XGB} / \mathrm{SHM}$, with groups being statistically different from one another from wk 12 to wk 23 (Fig. 2c). Body fat mass was also greater $(p<0.05)$ in $\mathrm{XGB} / \mathrm{OVX}$ mice than XGB/SHM mice at wk 12 and 18 (Fig. 2d). In S1, XGB did not result in any differences in mass of major adipose depots; however, total liver mass was reduced $(p=0.03)$ compared to baseline (Fig. 3a). In $\mathrm{S} 2, \mathrm{XGB} / \mathrm{OVX}$ mice exhibited greater subcutaneous $(p=0.02)$ and mesenteric $(p=0.04)$ adipose depot masses compared to $\mathrm{XGB} / \mathrm{SHM}$ mice at wk 12 , but not wk 18 or 24 (Fig. $3 \mathrm{~b})$. XGB resulted in reduced $(p=0.013)$ fasting serum TG concentrations after six wk compared to baseline in S1, but no differences in serum total
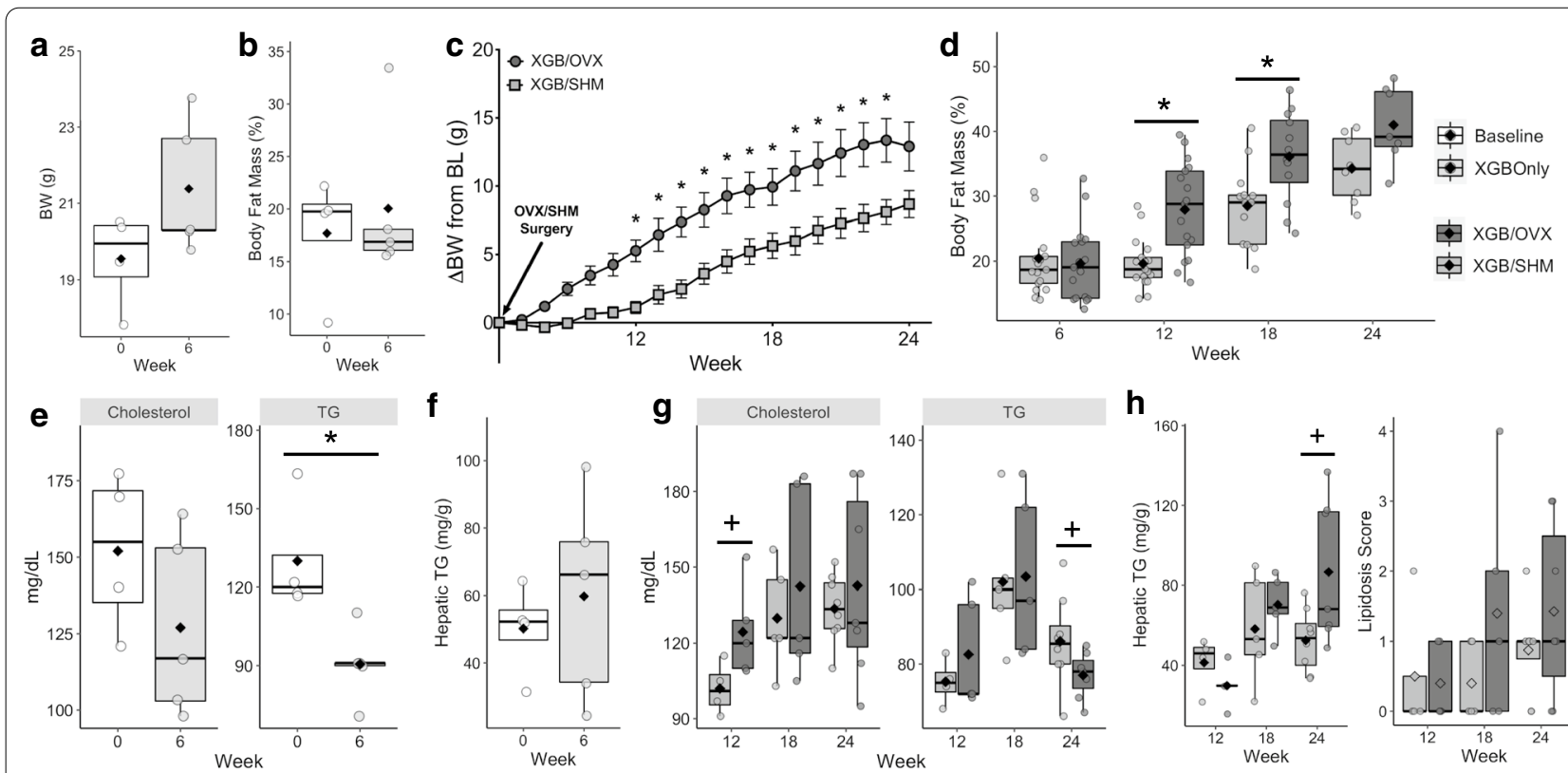

h

Fig. 2 Body weight (BW) (a, c), fat mass (b, d), blood lipids $(\mathbf{e}, \mathbf{g})$, and hepatic steatosis ( $\mathbf{f}, \mathbf{h})$ of mice undergoing cholecystectomy (XGB) only or XGB followed by either a sham (SHM) or ovariectomy (OVX) operation. In $\mathbf{a}, \mathbf{b}, \mathbf{e}, \mathbf{f}$ means are represented by a diamond, $w k 0, n=4 ; w k 6, n=5$. Data in panel $\mathbf{c}$ are expressed as LS means \pm SEM. In c, $\mathbf{d}$ wk 6-12, $n=17$ per group; wk 13-18, $n=12-13$ per group; wk 19-24, $n=7-8$ per group. In $\mathbf{g}, \mathbf{h}$ means are represented by a diamond, $w k 12, n=4-5$ per group; wk $18, n=5$ per group; $w k 24, n=7-8$ per group. Main effects of surgery are denoted by* $(p<0.05)$. Trends are denoted by $+(p<0.10)$. TG triglycerides 


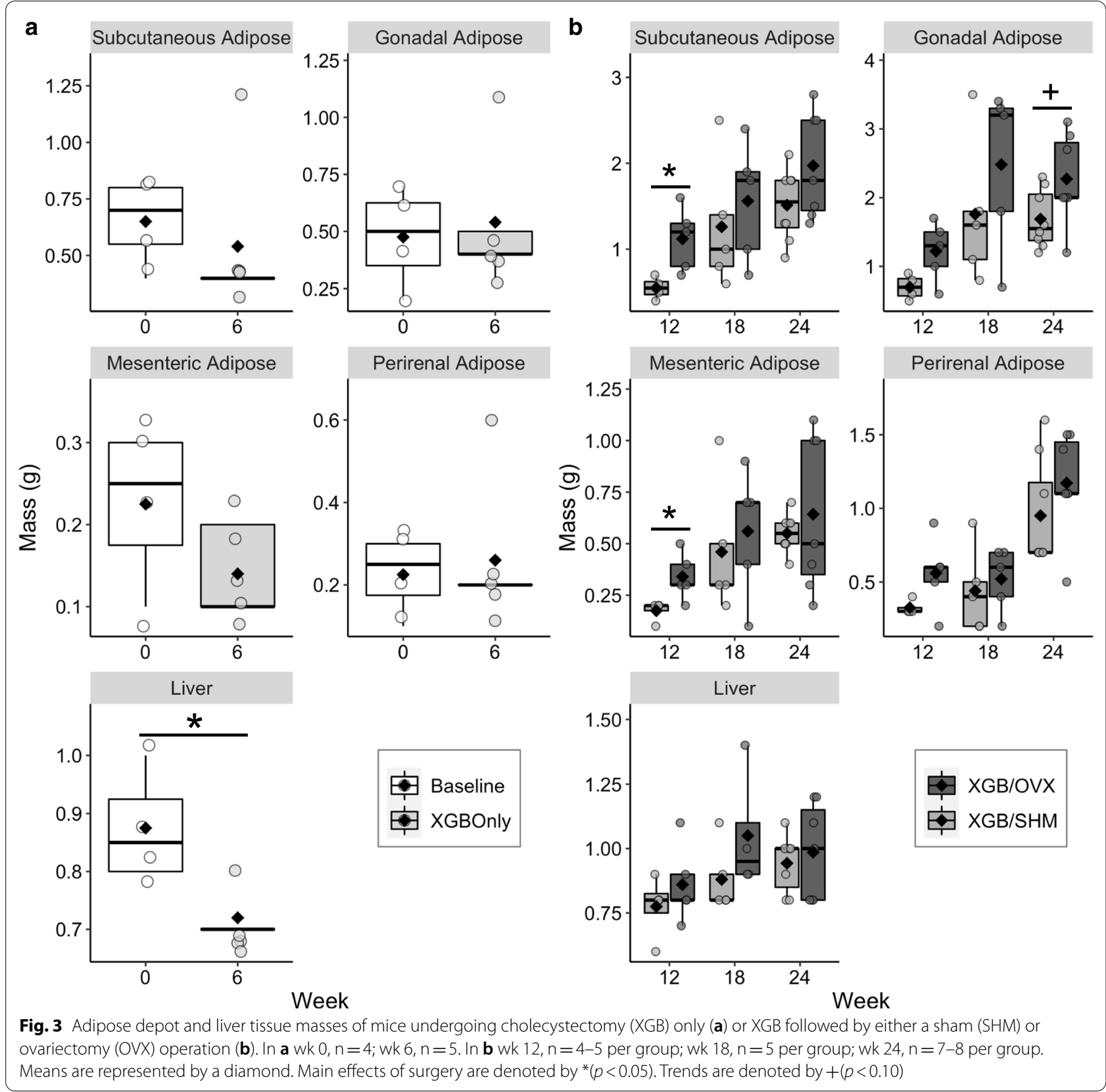

cholesterol concentrations were observed (Fig. 2e). Liver TG content was not altered six wk post-XGB (Fig. 2f). No consistent longitudinal patterns were observed in serum total cholesterol and TG concentrations between groups in S2 (Fig. 2g), likely due to a high degree of variation. At wk 12, serum total cholesterol concentrations tended to be greater in $\mathrm{XGB} / \mathrm{OVX}$ compared to $\mathrm{XGB} / \mathrm{SHM}$ $(p=0.068)$, but were not different at wk 18 and 24. Additionally, serum TG concentrations tended $(p=0.099)$ to be lower in $\mathrm{XGB} / \mathrm{OVX}$ compared to XGB/SHM at wk 24 , but not other timepoints. At wk 24, XGB/OVX mice tended to have greater hepatic TG content compared to $\mathrm{XGB} / \mathrm{SHM}$; however, hepatic lipidosis scoring revealed no differences between groups (Fig. $2 \mathrm{~h}$ ).

Expression of genes associated with BA signaling, synthesis, and transport, barrier function, and lipid metabolism were assessed in liver, ileal, and colonic tissues. In S1, hepatic relative expression of CYP7A1 (BA synthesis) was increased almost fivefold $(p=0.003)$, while relative expression of $H M G C R$ (cholesterol synthesis) was reduced more than fivefold $(p=0.0002)$ in XGB mice compared to baseline (Fig. 4a). Additionally, 


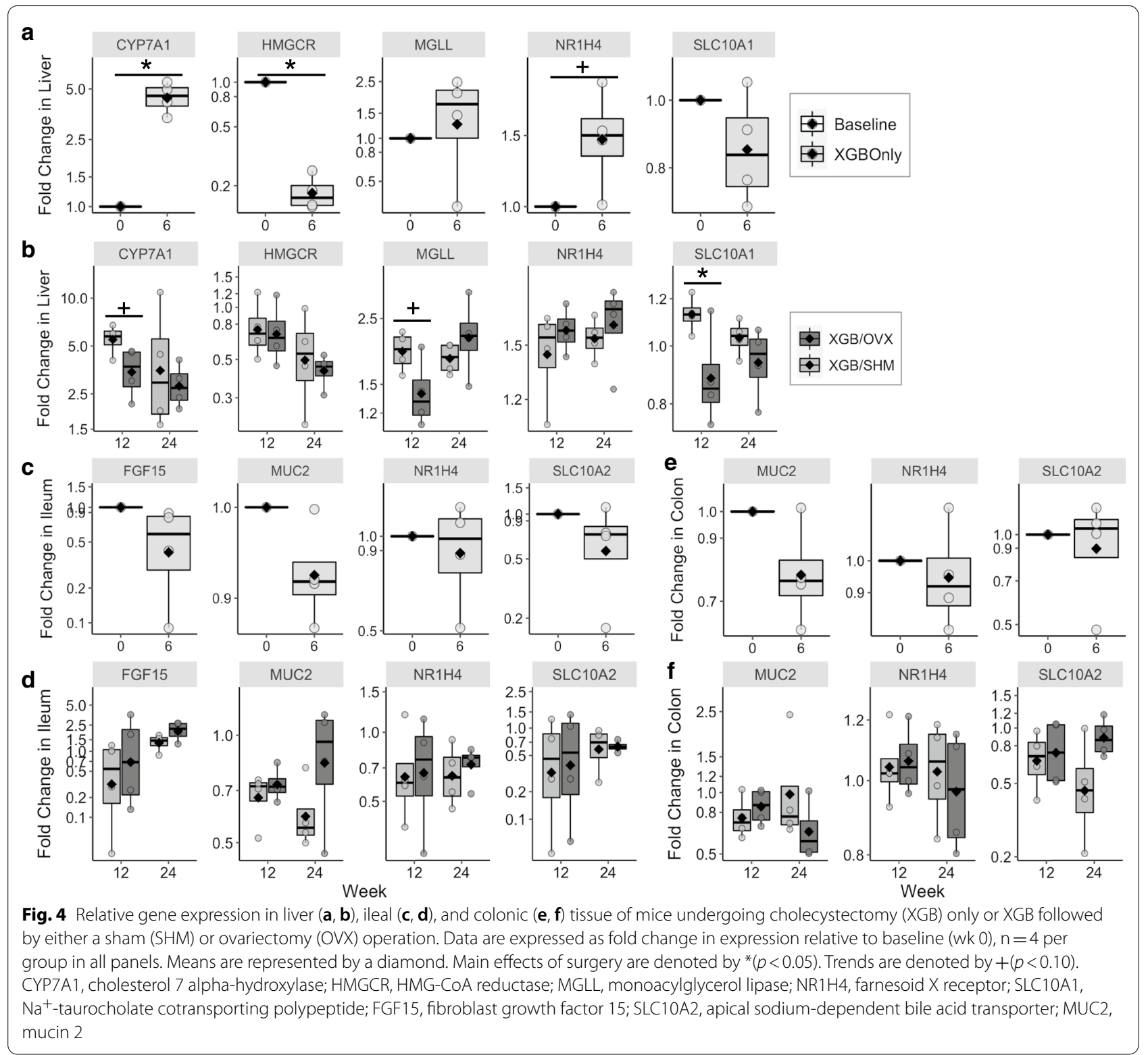

relative expression of $\mathrm{NR} 1 \mathrm{H} 4$ (FXR, major BA receptor) tended to be 1.5 -fold greater $(p=0.058)$ within six wk post-XGB compared to baseline. No differences were observed between XGB and baseline mice in the relative expression of hepatic $M G L L$ (monoacylglyceride catabolism) or SLC1OA1 (BA reabsorption). No differences in relative gene expression of FGF15 (FGF19 ortholog, feedback inhibition of BA synthesis, energy homeostasis), MUC2 (intestinal mucin), NR1H4, or SLC10A2 (BA reabsorption) were observed in ileal tissues (Fig. 4c) between baseline and six wk post-XGB. Additionally, no differences in relative gene expression of $M U C 2, N R 1 H 4$, or SLC1OA2 were observed in colonic tissues (Fig. 4e) between baseline and six wk post-XGB.

In liver tissues of S2, relative expression of SLC10A1 was lower $(p=0.048)$ in $\mathrm{XGB} / \mathrm{OVX}$ mice compared to $\mathrm{XGB} / \mathrm{SHM}$ at wk 12 but not different at wk 24 (Fig. 4b). Relative expression of CYP7A1 $(p=0.07)$ and $M G L L$ $(p=0.08)$ tended to be lower in XGB/OVX mice compared to XGB/SHM at wk 12, but were not different at wk 24. Relative expression of hepatic CYP7A1 and NR1H4 appeared to remain elevated and $H M G C R$ appeared to remain reduced in all S2 mice compared to S1 baseline mice, suggesting potentially chronic effects of XGB (Fig. 4). Similar to S1, there were no differences in 


\section{(See figure on next page.)}

Fig. 5 Alpha (a, d) and beta (b, c, e, f) diversity metrics of cecal (a-c) and fecal (d-f) microbiota of mice undergoing cholecystectomy (XGB) followed by either a sham (SHM) or ovariectomy (OVX) operation. Means in $\mathbf{a}, \mathbf{c}$ are represented by a diamond. In $\mathbf{a}-\mathbf{c}$, wk $12, n=4-5$ per group; wk $18, n=5$ per group; wk 24, $n=7-8$ per group. In $\mathbf{d}-\mathbf{f}, n=7-13$ per group. $\mathbf{b}$, e unweighted Unifrac distance principal coordinate analysis. $\mathbf{c}, \mathbf{f}$ weighted Unifrac distance principal coordinate analysis. In $\mathbf{b}, \mathbf{c}, \mathbf{e}, \mathbf{f}$, each dot represents a single sample. Larger dots are specific to wk 24 . Main effects of surgery are denoted by ${ }^{*}(p<0.05)$. Trends are denoted by $+(p<0.10)$

relative gene expression observed in ileal or colonic tissues (Fig. 4d, f) between XGB/OVX and XGB/SHM mice at either time point.

Diversity and composition of cecal and fecal microbial samples were assessed in S2 mice. Cecal microbial OTU richness $(p=0.03)$ and Shannon's diversity index $(p=0.03)$ were lower in XGB/OVX mice at wk 24 compared to XGB/SHM (Fig. 5a). Faith's phylogenetic diversity tended $(p=0.058)$ to be lower in XGB/OVX mice at wk 24 compared to XGB/SHM. No differences in cecal beta diversity as measured by unweighted (Fig. 5b) or weighted (Fig. 5c) Unifrac distances were observed between $\mathrm{XGB} / \mathrm{OVX}$ and $\mathrm{XGB} / \mathrm{SHM}$ mice at any time point, and no distinct clustering was observed in PCoA plots. In fecal samples, no differences in any alpha diversity metrics were observed between XGB/OVX and XGB/SHM mice at any time point (Fig. 5d). Similarly, no differences in beta diversity as measured by unweighted (Fig. 5e) or weighted (Fig. 5f) Unifrac distances were observed. Visualization of the 25 most abundant genera in both cecal and fecal samples demonstrated similar compositions between XGB/OVX and XGB/SHM mice at the end of S2 (Fig. 6). A few individual genera were observed to be differentially abundant. In cecal samples, relative abundance of Ruminococcaceae UCG-010 was lower $(p=0.03)$ in $\mathrm{XGB} / \mathrm{OVX}$ compared to $\mathrm{XGB} / \mathrm{SHM}$ mice (Fig. 7a). Additionally, relative abundance of cecal Parvibacter tended $(p=0.07)$ to be lower and Ruminococcaceae NK4A214 group tended $(p=0.07)$ to be greater in $\mathrm{XGB} / \mathrm{OVX}$ compared to XGB/SHM mice. In fecal samples, $\mathrm{XGB} / \mathrm{OVX}$ mice tended to have greater relative abundances of Lachnospiraceae UCG-006 $(p=0.08)$ and Ruminiclostridium $9(p=0.06)$ compared to XGB/SHM mice (Fig. 7b).

\section{Discussion}

In clinical trials, XGB has been shown to result in increased weight gain within six mo post-surgery [43]. Interestingly, within $3 \mathrm{yr}$ of XGB, females, but not males, were reported to have increased BMI compared to preop [44]. Pre-clinical XGB studies with male mice have not reported increased weight gain compared to SHM controls, suggesting there may be sex-specific responses to surgery $[30,31,45]$. In the present study, no changes in BW, body fat mass, or adipose depot masses were observed within six wk of XGB compared to baseline, but a non-XGB age-matched control was not utilized. Although clinical research has demonstrated an association between XGB and hepatic steatosis, no difference in hepatic TG was observed between XGB and baseline mice in this study [13-17]. Development of steatosis due to XGB may take longer than six wk, thus further preclinical research in females utilizing age-matched, intactgallbladder controls is needed.

Human menopause is associated with increased weight gain, ectopic fat deposition, and increased incidence of metabolic syndrome [35]. The rodent OVX model results in a similar phenotype. Previous studies in OVX rats and mice have demonstrated an increase in BW, fat mass, and hepatic TG content and lipidosis compared to intact controls [46-49]. However, it is not known if OVX results in a similar phenotype in XGB mice as in those with an intact gallbladder. A greater gain in total BW, particularly body fat mass, was observed in XGB/OVX mice compared to $\mathrm{XGB} / \mathrm{SHM}$ mice. Increased fat mass tended to be predominantly in gonadal fat pads by wk 24 , which is consistent with previous findings [49]. Interestingly, the difference in BW gain between XGB/OVX and $\mathrm{XGB} / \mathrm{SHM}$ groups appears to plateau around wk 14-15, potentially suggesting a delayed onset of rapid weight gain due to XGB that is accelerated by OVX. Hepatic TG only tended to be higher in XGB/OVX compared to $\mathrm{XGB} / \mathrm{SHM}$ by the end of the study. Over time, hepatic TG content increased in all S2 mice, which was likely due to a combination of aging and long-term consumption of a HFD, but potentially influenced by the XGB procedures at baseline. Previous studies in OVX mice and rats have also shown that OVX results in increased fasting LDL concentrations, with no consistent changes in fasting total cholesterol or TG observed [46, 49]. The present results were consistent with these findings in that no distinct patterns were observed in fasting total cholesterol or TG concentrations over time, however, LDL was not measured. In the present study, OVX was performed following XGB to mimic progression into menopause. Future studies would be useful to determine if timing of OVX, before or after XGB, differentially effects these outcomes.

Interestingly, there were no observed differences in relative expression of genes related to energy metabolism, gut integrity, or BA metabolism and transport in the ileum or colon between groups in both S1 or S2. In 


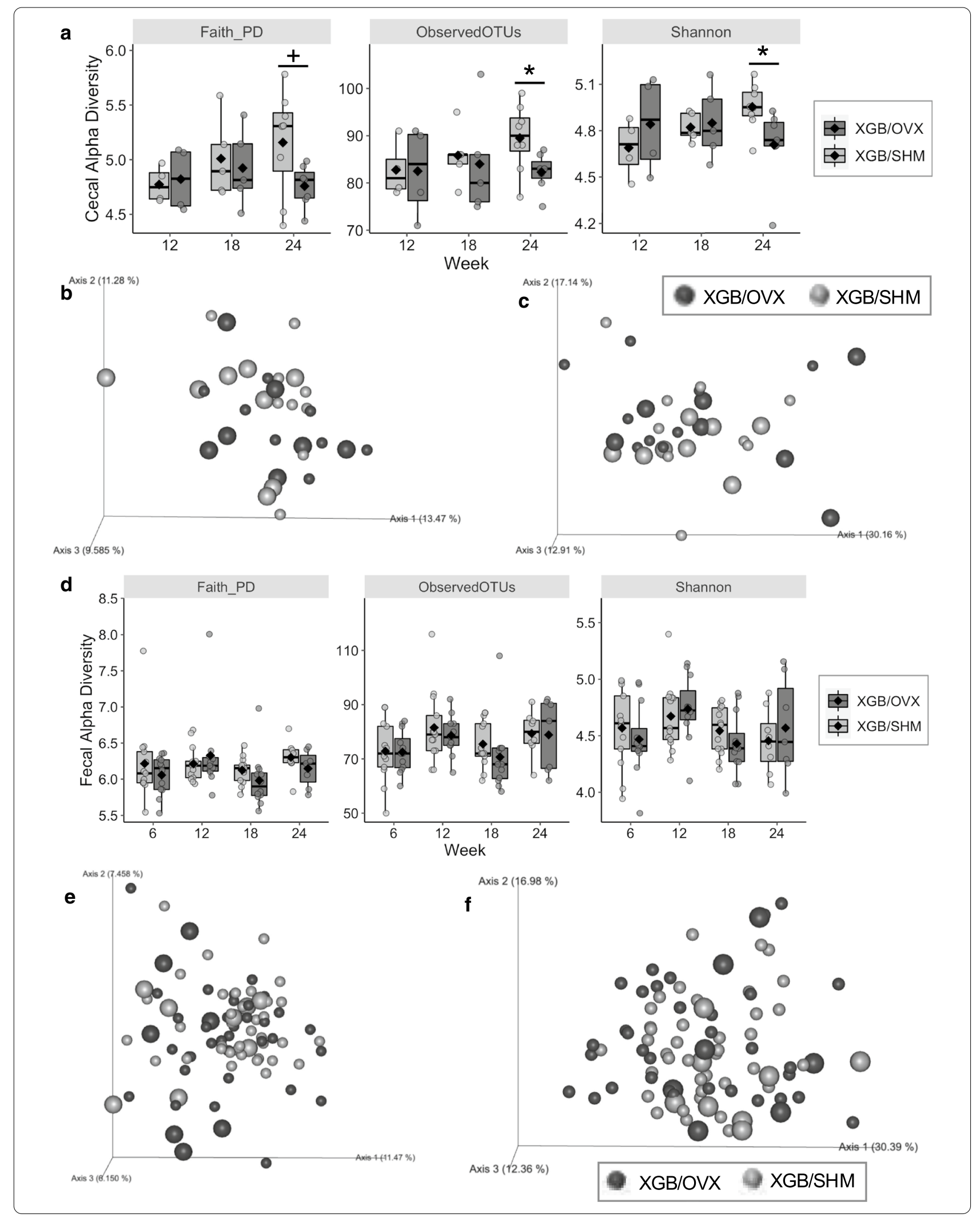


a $100-$

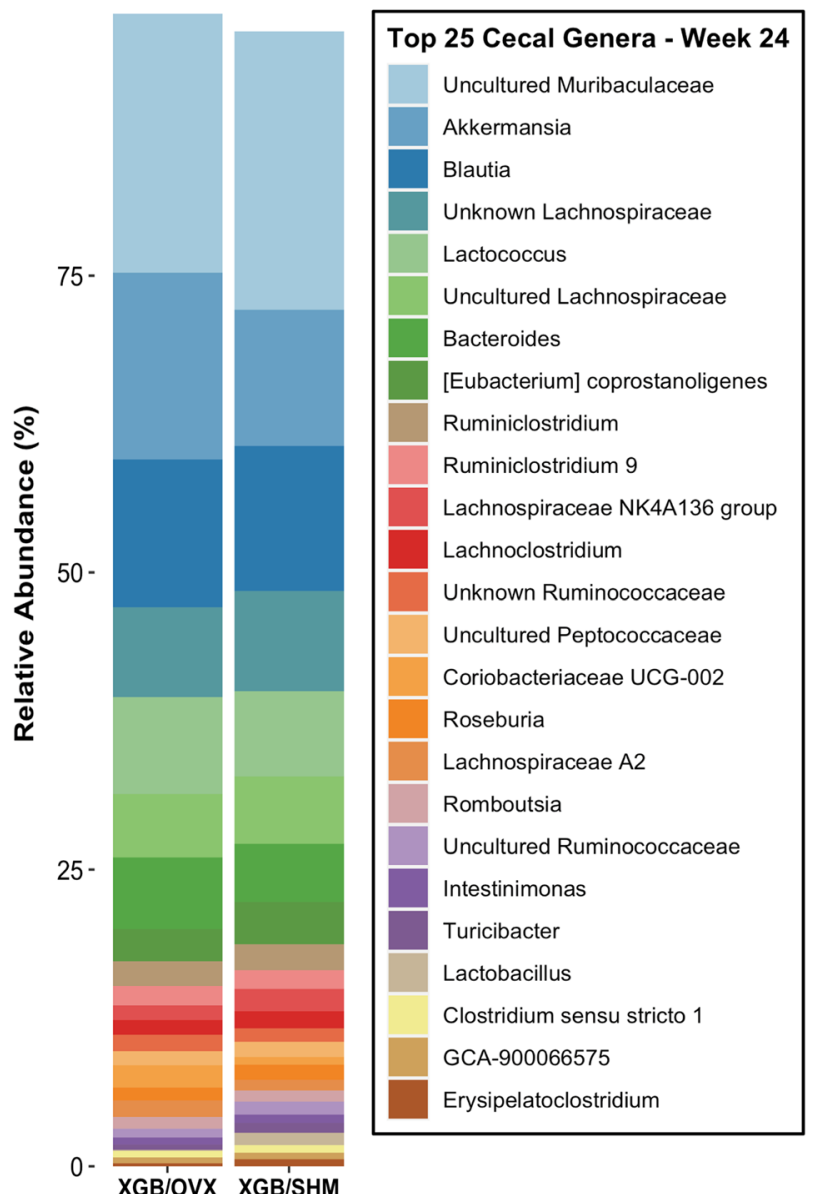

b 100 -

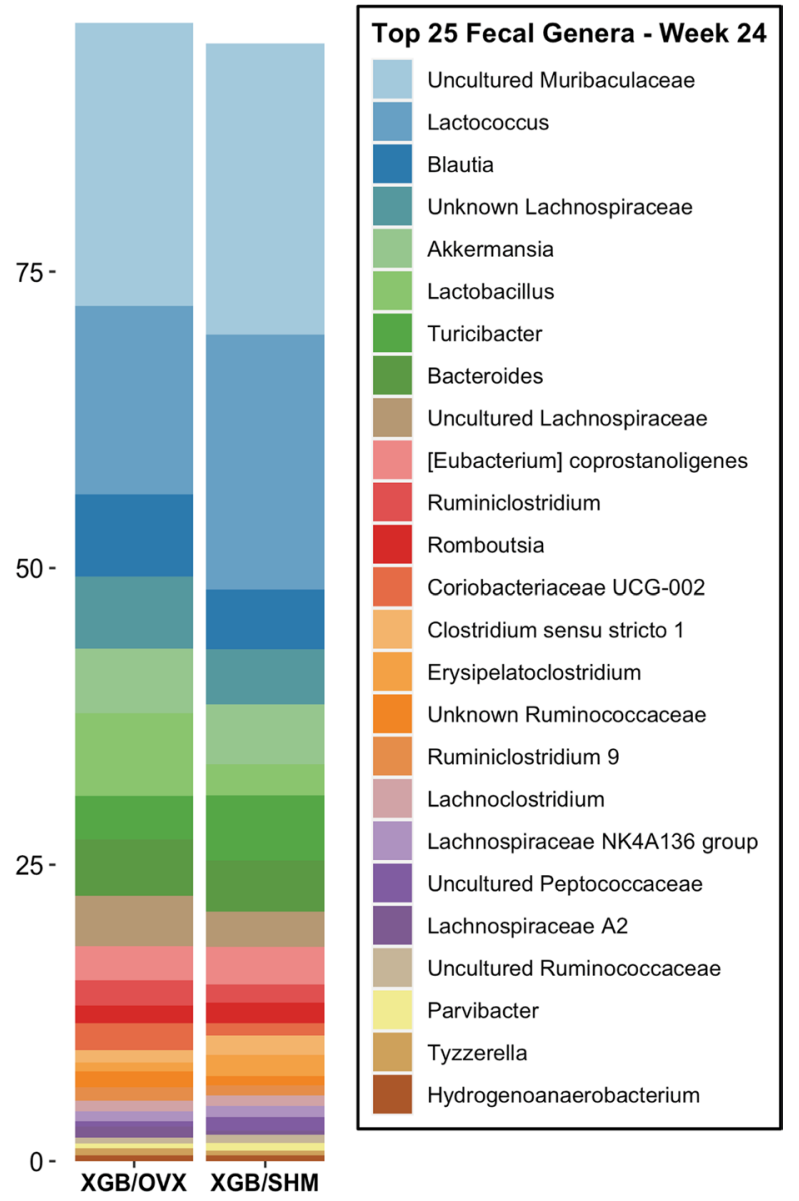

Fig. 6 Most abundant microbial genera at wk 24 in cecal and fecal samples of mice undergoing cholecystectomy (XGB) and followed by either a sham (SHM) or ovariectomy (OVX) operation. Data are expressed as means. In both samples, $n=7-8$ per group

S1, hepatic relative expression of CYP7A1 was increased almost fivefold while relative expression of $H M G C R$ was decreased more than fivefold, suggesting a shift away from endogenous cholesterol synthesis toward BA synthesis. In contrast, previous research in male XGB mice has not measured $H M G C R$ expression, but reported that hepatic CYP7A1 relative expression either does not change two-wk post-XGB [2] or decreases five-wk postXGB [30] compared to SHM controls. While the effects in the present study could be influenced by aging, the response is likely to be, at least in part, due to XGB and/ or chronic HFD consumption, as these genes encode proteins involved in lipid and BA metabolism. In nonXGB C57BL/6 mice, females are reported to have greater hepatic CYP7A1 expression compared to males [50]. Sexually dimorphic expression of CYP7A1 prior to XGB may explain contrasting results with previous research. Additionally, previous research demonstrated that female C57BL/6 mice fed a HFD $(60 \% \mathrm{kcal})$ had dramatically increased hepatic CYP7A1 expression and no change in $H M G C R$ expression compared to low-FD (16\% kcal) controls after $9 \mathrm{wk}$ [51]. In contrast again with the present findings, a study in male mice demonstrated that after five-wk post-XGB, ileal, but not hepatic, relative expression of NR1H4 was increased compared to controls. These findings collectively suggest there may be sex-specific effects of XGB on host health that have yet to be elucidated, and reinforce the critical need for detailed research in female XGB mice. Previous work from our lab has demonstrated that OVX in C57BL6 mice results in a number of changes in gene expression, including an increase in hepatic expression of $M G L L$ and a decrease in cecal expression of NR1H4 and FGF15 (unpublished). Such findings were not observed in S2-XGB/OVX did not influence relative expression of $\mathrm{NR} 1 \mathrm{H} 4$ in any tissues compared to XGB/SHM. Relative expression of hepatic CYP7A1, MGLL, and SLC1OA1 tended to be lower in $\mathrm{XGB} / \mathrm{OVX}$ mice at wk 12, but this effect was no longer 

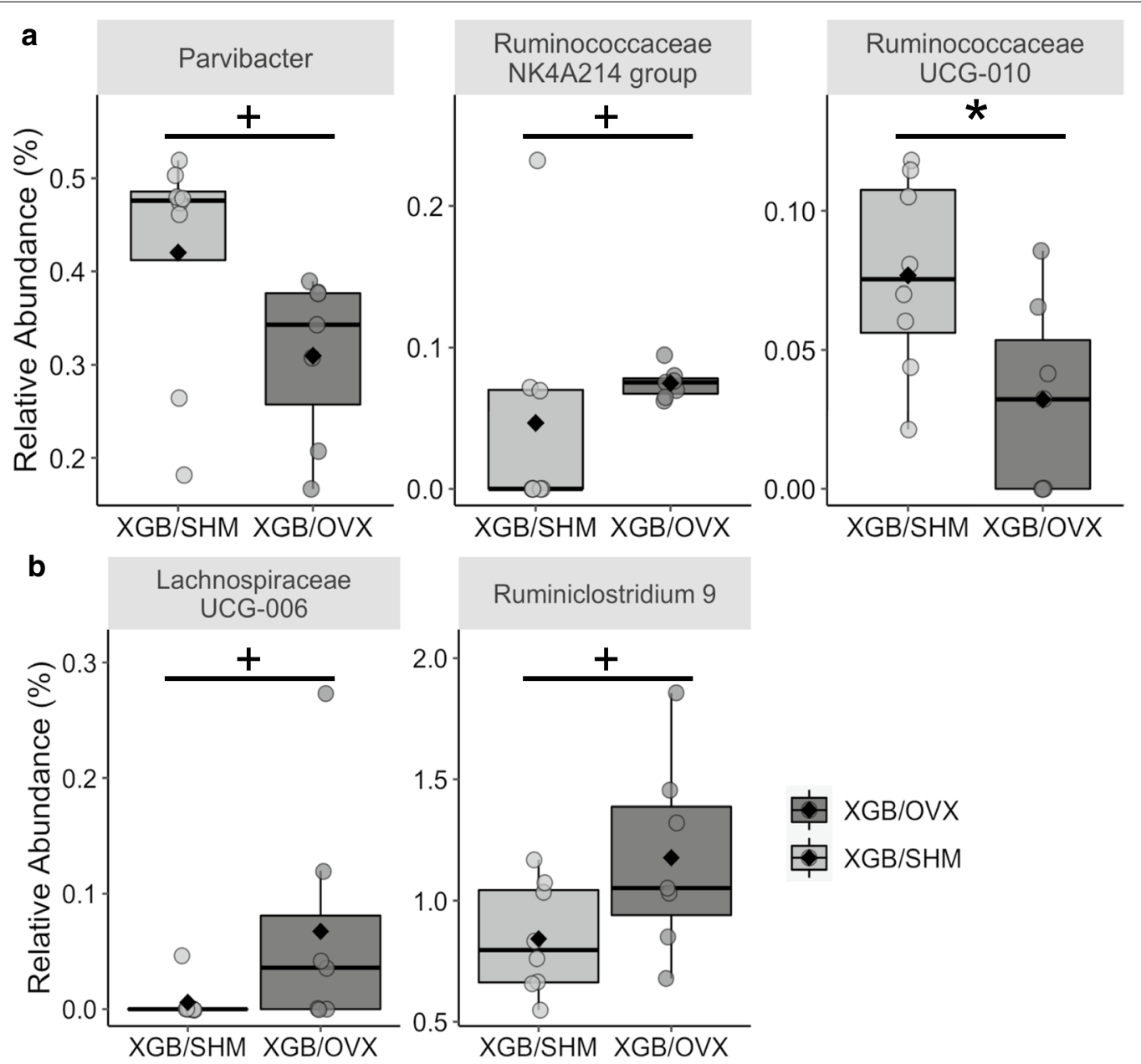

Fig. 7 Differentially abundant taxa of cecal (a) and fecal (b) samples at wk 24 of mice undergoing cholecystectomy (XGB) followed by either a sham $(\mathrm{SHM})$ or ovariectomy (OVX) operation. In all panels, $n=7-8$ per group. Means are represented by a diamond. Main effects of surgery are denoted by ${ }^{*}(p<0.05)$. Trends are denoted by $+(p<0.10)$

observed at wk 24. XGB may influence the expression of similar pathways, potentially masking differences due to OVX.

The present study did not observe any substantial effects of OVX on the diversity or composition of the cecal or fecal microbiota in XGB mice. By the end of the study, cecal alpha diversity metrics were lower in XGB/OVX mice, but no effect on beta diversity was observed. While one study has demonstrated that OVX may affect beta diversity [52], previous studies in our lab suggest no effect of OVX on alpha or beta diversity in mice fed a HFD or in rats fed a LFD (45, and unpublished). Additionally, there were no dramatic differences in relative abundance of individual genera in cecal and fecal samples. A few genera were found to be differentially abundant, specifically Ruminococcaceae UCG-010, Parvibacter, Ruminococcaceae NK4A214 group, Lachnospiraceae UCG-006, and Ruminiclostridium 9, but the physiological significance of these shifts is unknown. In contrast, previous studies in rats and mice have shown that OVX may increase relative abundance of Akkermansia, Anaerovibrio, Bifidobacterium animalis, Desulfovibrio, Dorea, and Lactobacillus, and decrease relative abundance of Ruminococcus and SMB53 [46, 52]. It is possible that baseline XGB may influence the microbiota composition and mask the effects of OVX. More research is needed to understand the independent effects of XGB on the microbiome.

\section{Conclusions}

In summary, we developed and demonstrated the feasibility of a novel model of XGB and subsequent OVX to best represent the main clinical population undergoing cholecystectomy. Furthermore, our pilot study demonstrated that in some ways, as in increased BW and fat mass, OVX resulted in the expected phenotype in 
previously XGB mice. However, the lack of other previously reported changes may be due to small sample size or potential metabolic alterations due to XGB. Future research utilizing our model is needed to elucidate the independent effects of XGB in female mice due to its translational and clinical potential.

\section{Abbreviations \\ BA: Bile acid; BW: Body weight; d: Day; Gl: Gastrointestinal; mo: Month; OVX: Ovariectomy; PCOA: Principal coordinate analysis; SEM: Standard error of the mean; SHM: Sham; SQ: Subcutaneous; TG: Triglyceride; wk: Week; XGB: Cholecystectomy; y: Year.}

\section{Acknowledgements}

Not applicable.

\section{Authors' contributions}

C.A., J.M.R., E.R.N., and K.S.S. planned the study. C.A., T-W.L.C., A.H.L., L.K.L., and M.D.V. conducted the study and collected data. C.A. interpreted data and drafted the manuscript. All authors read and approved the final manuscript.

\section{Funding}

This work was funded by USDA Hatch Grant ILLU-538-937.

\section{Availability of data and materials}

Datasets used in the present study are available from the corresponding authors upon request.

\section{Ethics approval}

All procedures were approved by the University of Illinois Institutional Animal Care and Use Committee before the study (protocol \#17040) and were performed in accordance with the United States Public Health Service Policy on Humane Care and Use of Laboratory Animals.

\section{Consent for publication}

Not applicable.

\section{Competing interests}

The authors declare no conflicts of interest.

\section{Author details}

1 Division of Nutritional Sciences, University of Illinois at Urbana-Champaign, 1207 W Gregory Dr, Urbana, IL 61801, USA. ${ }^{2}$ Department of Animal Sciences, University of Illinois at Urbana-Champaign, Urbana, IL, USA. ${ }^{3}$ College of Veterinary Medicine, University of Illinois at Urbana-Champaign, Urbana, IL, USA ${ }^{4}$ Cancer Center at Illinois, University of Illinois at Urbana-Champaign, Urbana, IL, USA. ${ }^{5}$ Carl R. Woese Institute for Genomic Biology, Anticancer Discovery from Pets to People Theme, University of Illinois Urbana-Champaign, IL, Urbana, USA. ${ }^{6}$ Department of Molecular and Integrative Physiology, University of Illinois at Urbana-Champaign, Urbana, IL, USA. ${ }^{7}$ University of Illinois Cancer Center, University of Illinois at Chicago, Chicago, IL, USA.

\section{Received: 18 November 2020 Accepted: 20 January 2021}

Published online: 11 February 2021

\section{References}

1. Lamberts MP, Kievit W, Özdemir C, Westert GP, Van Laarhoven CJHM, Drenth JPH. Value of EGD in patients referred for cholecystectomy: a systematic review and meta-analysis. Gastrointest Endosc. 2015;82:24-31.

2. Zhang F, Qin H, Zhao Y, Wei Y, Xi L, Rao Z, et al. Effect of cholecystectomy on bile acids as well as relevant enzymes and transporters in mice: Implication for pharmacokinetic changes of rifampicin. Eur J Pharm Sci. 2017:96:141-53.

3. Ingraham AM, Cohen ME, Ko CY, Hall BL. A current profile and assessment of north American cholecystectomy: results from the American college of surgeons national surgical quality improvement program. J Am Coll Surg. 2010;211:176-86.

4. de Bari O, Wang TY, Liu M, Portincasa P, Wang DQ-H. Estrogen induces two distinct cholesterol crystallization pathways by activating ERa and GPR30 in female mice. J Lipid Res. 2015;56:1691-700.

5. Mamdani MM, Tu K, Van Walraven C, Austin PC, David NC. Postmenopausal estrogen replacement therapy and increased rates of cholecystectomy and appendectomy. CMAJ. 2000;162:1421-4.

6. Racine A, Bijon A, Fournier A, Mesrine S, Clavel-Chapelon F, Carbonnel F, et al. Menopausal hormone therapy and risk of cholecystectomy: a prospective study based on the French E3N cohort. CMAJ. 2013;185:549-50.

7. Liu B, Beral V, Balkwill A, Green J, Sweetland S, Reeves G. Gallbladder disease and use of transdermal versus oral hormone replacement therapy in postmenopausal women: prospective cohort study. BMJ. 2008;337:a386.

8. Krondl A, Vavrinkova H, Michalec C. Effect of cholecystectomy on the role of the gall bladder in fat absorption. Gut. 1964;5:607-10.

9. de Menezes HL, Fireman PA, Wanderley VE, de Menconça AMMC, Bispo RKDA, Reis MR. Randomized study for assessment of hypolipidic diet in digestive symptoms immediately following laparoscopic cholecystectomy. Rev do Colégio Bras Cir. 2012;40:203-7.

10. Ure BM, Troidl $H$, Spangenberger W, Lefering R, Dietrich A, Eypasch EP, et al. Long-term results after laparoscopic cholecystectomy. Br J Surg. 1995;82:267-70.

11. Konsten J, Gouma DJ, von Meyenfeldt MF, Menheere P. Long-term followup after open cholecystectomy. Br J Surg. 1993;80:100-2.

12. Shen C, Wu X, Xu C, Yu C, Chen P, Li Y. Association of cholecystectomy with metabolic syndrome in a Chinese population. PLoS ONE. 2014;9:1-5.

13. Kwak M-S, Kim D, Chung GE, Kim W, Kim YJ, Yoon J-H. Cholecystectomy is independently associated with nonalcoholic fatty liver disease in an Asian population. World J Gastroenterol. 2015;21:6287-95.

14. Cortés V, Quezada N, Uribe S, Arrese M, Nervi F. Effect of cholecystectomy on hepatic fat accumulation and insulin resistance in non-obese Hispanic patients: a pilot study. Lipids Health Dis. 2017;16:129.

15. Nervi F, Arrese M. Editorial: Cholecystectomy and NAFLD: Does gallbladder removal have metabolic consequences? Am J Gastroenterol. 2013;108:959-61.

16. Yun S, Choi D, Lee KG, Kim HJ, Kang B-K, Kim H, et al. Cholecystectomy causes ultrasound evidence of increased hepatic steatosis. World J Surg. 2016;40:1412-21

17. Yue W, Sun $X$, Du T. Cholecystectomy versus central obesity or insulin resistance in relation to the risk of nonalcoholic fatty liver disease: the third US National Health and Nutrition Examination Survey. BMC Endocr Disord. 2019;19:1-9.

18. Chavez-Tapia NC, Mac Kinney-Novelo I, Sifuentes-Renterla SE, TorresZavala M, Castro-Gastelum G, Sánchez-Lara K, et al. Association between cholecystectomy for gallstone disease and risk factors for cardiovascular disease. Ann Hepatol. 2012;11:85-9.

19. Chen Y-K, Yeh J-H, Lin C-L, Peng C-L, Sung F-C, Hwang I-M, et al. Cancer risk in patients with cholelithiasis and after cholecystectomy: a nationwide cohort study. J Gastroenterol. 2014;49:923-31.

20. Zhang Y, Liu H, Li L, Ai M, Gong Z, He Y, et al. Cholecystectomy can increase the risk of colorectal cancer: a meta-analysis of 10 cohort studies. PLOS ONE. 2017:12:1-17.

21. Zuccato E, Venturi M, Di Leo G, Colombo L, Bertolo C, Doldi SB, et al. Role of bile acids and metabolic activity of colonic bacteria in increased risk of colon cancer after cholecystectomy. Dig Dis Sci. 1993;38:514-9.

22. Liu Y, He Y, Li T, Xie L, Wang J, Qin X, et al. Risk of primary liver cancer associated with gallstones and cholecystectomy: a meta-analysis. PLoS ONE. 2014;9:e109733.

23. Sung HJ, Paik CN, Chung WC, Lee KM, Yang JM, Choi MG. Small intestinal bacterial overgrowth diagnosed by glucose hydrogen breath test in postcholecystectomy patients. J Neurogastroenterol Motil. 2015;21:545-51.

24. Kim DB, Paik C-N, Kim YJ, Lee JM, Jun K-H, Chung WC, et al. Positive glucose breath tests in patients with hysterectomy, gastrectomy, and cholecystectomy. Gut Liver. 2017;11:237-42.

25. Mercan E, Duman U, Tihan D, Dilektasli E, Senol K. Cholecystectomy and duodenogastric reflux: interacting effects over the gastric mucosa. Springerplus. 2016;5:1970.

26. Yoon W, Kim H-N, Park E, Ryu S, Chang Y, Shin H, et al. The impact of cholecystectomy on the gut microbiota: a case-control study. J Clin Med. 2019;8:79. 
27. Kang Z, Lu M, Jiang M, Zhou D, Huang H. Proteobacteria acts as a pathogenic risk-factor for chronic abdominal pain and diarrhea in postcholecystectomy syndrome patients: a gut microbiome metabolomics study. Med Sci Monit. 2019;25:7312-20.

28. Keren N, Konikoff FM, Paitan Y, Gabay G, Reshef L, Naftali T, et al. Interactions between the intestinal microbiota and bile acids in gallstones patients. Environ Microbiol Rep. 2015;7:874-80.

29. Wang W, Wang J, Li J, Yan P, Jin Y, Zhang R, et al. Cholecystectomy damages aging-associated intestinal microbiota construction. Front Microbiol. 2018;9:1402.

30. Amigo L, Husche C, Zanlungo S, Lütjohann D, Arrese M, Miquel JF, et al. Cholecystectomy increases hepatic triglyceride content and very-lowdensity lipoproteins production in mice. Liver Int. 2011;31:52-64.

31. Cortés V, Amigo L, Zanlungo S, Galgani J, Robledo F, Arrese M, et al. Metabolic effects of cholecystectomy: gallbladder ablation increases basal metabolic rate through G-protein Coupled Bile acid Receptor Gpbar1dependent mechanisms in mice. PLoS ONE. 2015;10:1-15.

32. Lefebvre P, Cariou B, Lien F, Kuipers F, Staels B. Role of bile acids and bile acid receptors in metabolic regulation. Physiol Rev. 2009;89:147-91.

33. Long SL, Gahan CGM, Joyce SA. Interactions between gut bacteria and bile in health and disease. Mol Aspects Med. 2017;56:54-65.

34. Haro C, Rangel-Zúñiga OA, Alcalá-Díaz JF, Gómez-Delgado F, PérezMartínez P, Delgado-Lista J, et al. Intestinal microbiota is influenced by gender and body mass index. PLoS ONE. 2016;11:e0154090.

35. Stefanska A, Bergmann K, Sypniewska G. Metabolic syndrome and menopause: pathophysiology, clinical and diagnostic significance. Adv Clin Chem. 2015;72:1-75.

36. Rector RS, Thyfault JP, Morris RT, Laye MJ, Borengasser SJ, Booth FW, et al Daily exercise increases hepatic fatty acid oxidation and prevents steatosis in Otsuka Long-Evans Tokushima Fatty rats. Am J Physiol Gastrointest Liver Physiol. 2008;294:619-26.

37. Alexander C, Cross T-WL, Devendran S, Neumer F, Theis S, Ridlon JM, et al. Effects of prebiotic inulin-type fructans on blood metabolite and hormone concentrations and faecal microbiota and metabolites in overweight dogs. Br J Nutr. 2018;120:711-20.

38. Caporaso JG, Lauber CL, Walters WA, Berg-Lyons D, Huntley J, Fierer $\mathrm{N}$, et al. Ultra-high-throughput microbial community analysis on the Illumina HiSeq and MiSeq platforms. ISME J. 2012;6:1621-4.

39. Bolyen E, Rideout JR, Dillon MR, Bokulich NA, Abnet CC, Al-Ghalith GA, et al. Reproducible, interactive, scalable and extensible microbiome data science using QIIME 2. Nat Biotechnol. 2019;37:852-7.

40. Callahan BJ, McMurdie PJ, Rosen MJ, Han AW, Johnson AJA, Holmes SP. DADA2: High-resolution sample inference from Illumina amplicon data. Nat Methods. 2016;13:581-3.
41. Quast C, Pruesse E, Yilmaz P, Gerken J, Schweer T, Yarza P, et al. The SILVA ribosomal RNA gene database project: Improved data processing and web-based tools. Nucleic Acids Res. 2013:41:D590-6.

42. Lozupone C, Knight R. UniFrac : a new phylogenetic method for comparing microbial communities. Appl Environ Microbiol. 2005;71:8228-35.

43. Yazdankhah Kenary A, Yaghoobi Notash A, Nazari M, Yaghoobi Notash A, Borjian A, Afshin N, et al. Measuring the rate of weight gain and the influential role of diet in patients undergoing elective laparoscopic cholecystectomy: a 6-month follow-up study. Int J Food Sci Nutr. 2012;63:645-8.

44. Ali R, Cahill R, Watson R. Weight gain after laparoscopic cholecystectomy. Ir J Med Sci. 2004;173:9-12.

45. Rodriguez PM, Cruz NI, Gonzalez Cl, Lopez R. The effect of a high fat diet on the incidence of colonic cancer after cholecystectomy in mice. Cancer. 1988;62:727-9.

46. Cross T-WL, Zidon TM, Welly RJ, Park Y-M, Britton SL, Koch LG, et al. Soy improves cardiometabolic health and cecal microbiota in female low-fit rats. Sci Rep. 2017;7:9261.

47. Hong J, Stubbins RE, Smith RR, Harvey AE, Núñez NP. Differential susceptibility to obesity between male, female and ovariectomized female mice. Nutr J. 2009;8:1-5.

48. Quinn MA, Xu X, Ronfani M, Cidlowski JA. Estrogen deficiency promotes hepatic steatosis via a glucocorticoid receptor-dependent mechanism in mice. Cell Rep. 2018;22:2690-701.

49. Rogers NH, Li JWP, Strissel KJ, Obin MS, Greenberg AS. Reduced energy expenditure and increased inflammation are early events in the development of ovariectomy-induced obesity. Endocrinology. 2009;150:2161-8.

50. Wankhade UD, Zhong Y, Kang P, Alfaro M, Chintapalli SV, Piccolo BD, et al. Maternal high-fat diet programs offspring liver steatosis in a sexually dimorphic manner in association with changes in gut microbial ecology in mice. Sci Rep. 2018;8:1-15.

51. Arisqueta L, Navarro-Imaz H, Labiano I, Rueda Y, Fresnedo O. High-fat diet overfeeding promotes nondetrimental liver steatosis in female mice. Am J Physiol Gastrointest Liver Physiol. 2018;315:G772-80.

52. Choi S, Hwang Y-J, Shin M-J, Yi H. Difference in the gut microbiome between ovariectomy-induced obesity and diet-induced obesity. J Microbiol Biotechnol. 2017;27:2228-36.

\section{Publisher's Note}

Springer Nature remains neutral with regard to jurisdictional claims in published maps and institutional affiliations.
Ready to submit your research? Choose BMC and benefit from:

- fast, convenient online submission

- thorough peer review by experienced researchers in your field

- rapid publication on acceptance

- support for research data, including large and complex data types

- gold Open Access which fosters wider collaboration and increased citations

- maximum visibility for your research: over $100 \mathrm{M}$ website views per year

At BMC, research is always in progress.

Learn more biomedcentral.com/submissions 